\title{
Loss of switch/sucrose non-fermenting complex protein expression is associated with dedifferentiation in endometrial carcinomas
}

\author{
Anthony N Karnezis ${ }^{1,11}$, Lien N Hoang ${ }^{1,11}$, Mackenzie Coatham ${ }^{2,3}$, Sarah Ravn ${ }^{3}$,
} Noorah Almadani ${ }^{1}$, Basile Tessier-Cloutier ${ }^{1}$, Julie Irving ${ }^{1,4}$, Bo Meng ${ }^{5}$, Xiaodong Li ${ }^{5}$, Christine Chow ${ }^{1}$, Jessica McAlpine ${ }^{6}$, Kuan-Ting Kuo ${ }^{7}$, Tsui-Lien Mao ${ }^{7}$, Bojana Djordjevic ${ }^{8}$, Robert A Soslow ${ }^{9}$, David G Huntsman ${ }^{1}$, C Blake Gilks ${ }^{1}$, Martin Köbel ${ }^{10}$ and Cheng-Han Lee ${ }^{5}$

${ }^{1}$ Department of Pathology and Laboratory Medicine, and Genetic Pathology Evaluation Center, Vancouver General Hospital and University of British Columbia, Vancouver, BC, Canada; ${ }^{2}$ Department of Oncology, University of Alberta, Edmonton, AB, Canada; ${ }^{3}$ Department of Obstetrics and Gynecology, University of Alberta, Edmonton, AB, Canada; ${ }^{4}$ Department of Laboratory Medicine, Pathology, and Medical Genetics, Royal Jubilee Hospital, Victoria, BC, Canada; ${ }^{5}$ Department of Laboratory Medicine and Pathology, Royal Alexandra Hospital and University of Alberta, Edmonton, AB, Canada; ${ }^{6}$ Department of Obstetrics and Gynecology, University of British Columbia, Vancouver, BC, Canada; ${ }^{7}$ Department of Pathology, National Taiwan University Hospital and College of Medicine, Taipei, Taiwan; ${ }^{8}$ Department of Pathology and Laboratory Medicine, The Ottawa Hospital, Ottawa, ON, Canada; ${ }^{9}$ Department of Pathology, Memorial Sloan Kettering Cancer Center, New York, NY, USA and ${ }^{10}$ Department of Pathology and Laboratory Medicine, Calgary Laboratory Services and University of Calgary, Calgary, AB, Canada

\begin{abstract}
Dedifferentiated endometrial carcinoma is an aggressive type of endometrial cancer that contains a mix of low-grade endometrioid and undifferentiated carcinoma components. We performed targeted sequencing of eight dedifferentiated carcinomas and identified somatic frameshift/nonsense mutations in SMARCA4, a core ATPase of the switch/sucrose non-fermenting (SWI/SNF) complex, in the undifferentiated components of four tumors. Immunohistochemical analysis confirmed the loss of SMARCA4 in the undifferentiated component of these four SMARCA4-mutated cases, whereas the corresponding low-grade endometrioid component showed retained SMARCA4 expression. An expanded survey of other members of the SWI/SNF complex showed SMARCB1 loss in the undifferentiated component of two SMARCA4-intact tumors, and all SMARCA4- or SMARCB1-deficient tumors showed concomitant loss of expression of SMARCA2. We subsequently examined the expression of SMARCA2, SMARCA4, and SMARCB1 in an additional set of 22 centrally reviewed dedifferentiated carcinomas and 31 grade 3 endometrioid carcinomas. Combining the results from the index and the expansion set, 15 of $30(50 \%)$ of the dedifferentiated carcinomas examined showed either concurrent SMARCA4 and SMARCA2 loss (37\%) or concurrent SMARCB1 and SMARCA2 loss (13\%) in the undifferentiated component. The loss of SMARCA4 or SMARCB1 was mutually exclusive. All 31 grade 3 endometrioid carcinomas showed intact expression of these core SWI/SNF proteins. The majority $(73 \%)$ of the SMARCA4/SMARCA2-deficient and half of SMARCB1/SMARCA2-deficient undifferentiated component developed in a mismatch repair-deficient molecular context. The observed spatial association between SWI/SNF protein loss and histologic dedifferentiation suggests that inactivation of these core SWI/SNF proteins may contribute to the development of dedifferentiated endometrial carcinoma.
\end{abstract}

Modern Pathology (2016) 29, 302-314; doi:10.1038/modpathol.2015.155; published online 8 January 2016

Correspondence: Dr C-H Lee, MD, PhD, Department of Laboratory Medicine and Pathology, Royal Alexandra Hospital and University of Alberta, Room DTC 5002-16, 10240 Kingsway, Edmonton, AB T5H 3V9, Canada.

E-mail: chlee2@ualberta.ca

${ }^{11}$ These authors contributed equally to this work.

Received 4 September 2015; accepted 20 November 2015; published online 8 January 2016
Dedifferentiated endometrial carcinoma is a subtype of endometrial cancer in which an endometrioid carcinoma occurs in association with an undifferentiated carcinoma. In the initial series described by Silva et $a l^{1,2}$ the endometrioid component displayed either FIGO (The International 
Federation of Gynecology and Obstetrics) grade 1 or 2 morphology. In contrast to the differentiated component, the undifferentiated component was comprised of monotonous medium to large round/polygonal cells growing in solid discohesive sheets, without any evidence of glandular formation. The undifferentiated component showed a high mitotic rate with the majority showing tumor necrosis. Some of the undifferentiated components also exhibited focal rhabdoid cytologic features, focal marked nuclear pleomorphism, or abrupt keratinization. It was postulated that the undifferentiated carcinoma represented a form of tumor progression termed 'dedifferentiation' from the pre-existing low-grade endometrioid carcinoma. This phenomenon of dedifferentiation was found to be associated with a highly aggressive clinical course, even in cases where the undifferentiated carcinoma constituted a minor component of the overall tumor. ${ }^{1}$

Since the original report by Silva et $a l_{,}^{1,2}$ dedifferentiated endometrial carcinoma has become increasingly recognized by pathologists. ${ }^{3-6}$ An association between dedifferentiated/undifferentiated histology and mismatch repair protein deficiency/ Lynch syndrome was subsequently recognized, ${ }^{7-9}$ with $58 \%$ of the dedifferentiated endometrial carcinomas reported to show mismatch repair deficiency by immunohistochemistry. ${ }^{5}$ In these mismatch repair-deficient cases, the differentiated endometrioid component and undifferentiated component both showed an identical pattern of mismatch repair deficiency. Most recently, Kuhn et $a l^{4}$ compared the mutation profiles between the endometrioid component and undifferentiated component of a series of dedifferentiated endometrial carcinomas. They identified the same somatic mutations in the corresponding endometrioid and undifferentiated components in all cases. Although these findings support a clonal origin between the differentiated endometrioid and the undifferentiated component of dedifferentiated endometrial carcinoma, the precise molecular mechanism underlying this postulated progression from low-grade endometrioid carcinoma to undifferentiated carcinoma (dedifferentiation) remains poorly understood.

In this study, we performed targeted sequencing and immunohistochemical analysis comparing the endometrioid and the undifferentiated component of a series of dedifferentiated endometrial carcinomas. We identified frequent coinactivation of SMARCA4/ SMARCA2 or SMARCB1/SMARCA2 in the undifferentiated component of these tumors, indicating that a loss of expression of these core switch/sucrose non-fermenting (SWI/SNF) complex proteins may contribute to the process of dedifferentiation in endometrial carcinoma.

\section{Materials and methods}

\section{Study Samples}

The study included 8 dedifferentiated endometrial carcinomas in the index series and 22 dedifferentiated endometrial carcinomas in the validation series. All of the endometrial carcinomas included in this study were from hysterectomy specimens. The cases from the index series were identified and obtained from the pathology archives at Calgary Laboratory Services (Calgary, AB, Canada) and Royal Alexandra Hospital (Edmonton, AB, Canada), whereas the cases from the validation series were identified and obtained from the pathology archives at Vancouver General Hospital (Vancouver, BC, Canada), Calgary Laboratory Services (Calgary, AB, Canada), Royal Alexandra Hospital (Edmonton, AB, Canada), The Ottawa Hospital (Ottawa, ON, Canada), and Memorial Sloan Kettering Cancer Center (New York, NY, USA). All cases were centrally reviewed (ANK, LH, and CHL), fulfilling the morphologic features described by Silva et al. ${ }^{1,2}$ We excluded cases that were inadequately fixed such that histologic evaluation could not be properly performed. Tumors showing focal glandular differentiation (away from apparent interface between differentiated and undifferentiated components), well-demarcated cohesive lobules, and/or cellular spindling (spindled epithelial component) were excluded. A series of 31 FIGO grade 3 endometrioid carcinomas that were initially diagnosed as dedifferentiated endometrial carcinomas but subsequently revised to FIGO grade 3 endometrioid carcinomas upon histologic review were included for comparison. The study was approved by the Institutional Review Board.

\section{DNA Extraction}

For each case, tissue cores $(0.6 \mathrm{~mm})$ of tumor (with the endometrioid and the undifferentiated component separately cored whenever possible) and the corresponding normal tissues were obtained from formalin-fixed, paraffin-embedded blocks. The tumor cores were obtained from areas showing the most histologically viable tumor with the least amount of stromal tissue. Normal tissue (ie, uterine cervix, fallopian tube, and ovary) that was distant from and uninvolved by endometrial carcinoma was used for comparison. DNA was extracted from the tissue cores using the Qiagen formalin-fixed, paraffin-embedded tissue DNA extraction kit based on the manufacturer's protocols.

\section{Targeted Gene Panel Sequencing Analysis and Validations}

We performed sequencing analysis to detect mutations in 26 genes that have been previously found to be recurrently mutated in carcinomas of the 
gynecologic tract. These included the full coding regions of $A K T 1, A R I D 1 A, F B X W 7, F G F R 2, J A K 1$, KRAS, MLH1, MSH2, MSH6, NRAS, PIK3CA, PIK3R1, PIK3R2, PMS2, POLE, PPP2R1A, PTEN, RNF43, RPL22, SMARCA4, STK11, SPOP, TP53, and in selected exons in FOXL2 (exon 1), CTNNB1 (exon 3), and BRAF (exon 15). ${ }^{10}$ The Illumina custom TruSeq amplicon panel was designed using Illumina's DesignStudio and included 1173 amplicons (175 bp) that covers $98 \%$ of the exons and untranslated regions of these 26 genes. Custom amplicon libraries were prepared starting with $250 \mathrm{ng}$ of DNA as per Ilumina's Custom TruSeq Library Preparation protocol. Before pooling, normalization was performed by quantifying individual libraries using the Qubit fluorometer, and then pooled based on equal concentrations. Library pools were then quantitated for amplifiable libraries using the Kapa Biosystems FAST qPCR SYBR Quantification Kit based on the manufacturer's protocols. Pooled TruSeq libraries were sequenced using the Illumina MiSeq using 300 Cycle V2 Kits. Analysis was performed using the MiSeq Reporter and somatic variant caller 3.2.3.0. Only nonsynonymous mutations passing quality filter with at least $10 \%$ variant allele frequency were further evaluated. These mutations were manually checked in bam files using Integrated Genome Viewer. Selected mutations (such as those involving SMARCA4) were validated orthogonally by direct Sanger sequencing using primer sets that target the regions containing the mutations; comparisons were made to the corresponding genomic sequences from normal tissue to determine the somatic/germline status of these mutations.

\section{Tissue Microarray Construction}

Two $0.6 \mathrm{~mm}$ cores from each dedifferentiated endometrial carcinoma were arrayed and placed on a single tissue microarray. Whenever possible, the different histologic components (endometrioid component and undifferentiated component) were separately cored.

\section{Immunohistochemistry and Interpretation}

SMARCA4 (BRG1) immunohistochemical analysis was performed on representative whole tissue sections from the hysterectomy specimens, whereas SMARCA2 (BRM), SMARCB1 (INI1), MLH1, MSH2, MSH6, and PMS2 immunohistochemical analyses were initially performed on tissue microarrays, followed by subsequent whole section analysis in cases with abnormal or equivocal results. For mismatch repair proteins (MLH1, MSH2, MSH6, and PMS2), the primary antibodies used and the staining methods are the same as that reported previously. ${ }^{10,11}$ The slides were incubated with MLH1 (Dako; clone ES05 1:100), MSH2 (NCL; clone
25D12 prediluted), MSH6 (BD Bioscience; 44/MSH6 1:2000), PMS2 (BD Bioscience; A16-4 1:100), and processed using the Leica Bond Max platform $q$ (Leica Microsystems, Wetzlar, Germany) as per the manufacturer's protocol with proprietary reagents. The detection system used was the Bond polymer refine. For SMARCA2, SMARCA4, and SMARCB1, the slides were incubated with SMARCA2/BRM (1:50, clone HPA029981; Sigma, St Louis, MO, USA), SMARCA4/BRG1 (1:25, clone EPNCIR111A, ab110641; Abcam, Toronto, ON, Canada), and SMARCB1/BAF47/INI1 (1:50，25/BAF47，612110; BD Biosciences, Mississauga, ON, USA), and processed using the Ventana Discovery XT, and the Ventana Benchmark XT and Benchmark Ultra automated systems (Ventana Medical Systems, Tucson, AZ, USA).

All whole sections and tissue microarrays were scored by two pathologists (ANK and CHL). The differentiated and undifferentiated components of all dedifferentiated carcinomas were scored separately. For SMARCA2, SMARCA4, and SMARCB1, tumors were scored as SMARCA2/SMARCA/SMARCB1intact if any tumor cell nuclei showed nuclear staining; tumors scored as positive usually showed diffuse, moderate to strong staining. ${ }^{12}$ Tumors were scored as SMARCA2/SMARCA4/SMARCB1-deficient if tumor nuclei showed no staining in the presence of internal positive control (nuclear staining of the stromal fibroblasts, endothelial cells, and inflammatory cells). For MLH1, MSH2, MSH6, and PMS2, staining was considered abnormal q (mismatch repair-deficient) when there was loss of nuclear expression by the tumor cells compared with internal positive control (nuclear staining of the stromal fibroblasts, endothelial cells, and inflammatory cells). ${ }^{10}$

\section{Results}

Targeted Sequencing Identifies Somatic-Inactivating SMARCA4 Mutations in the Undifferentiated Component

We performed targeted next-generation sequencing on an index series of 8 dedifferentiated endometrial carcinomas, with both the endometrioid and undifferentiated components analyzed in 4 cases and only the undifferentiated component analyzed in the remaining 4 cases because of limited material in the available blocks provided. There was an average of 1675-fold coverage per amplicon (range 1181- to 2657-fold) and $94 \%$ of the amplicons had a median coverage across all the samples of at least 50-fold. All eight dedifferentiated endometrial carcinomas contained a mix of low-grade (FIGO grade 1 or 2) endometrioid carcinoma and undifferentiated carcinoma (Table 1 and Figure 1). The undifferentiated component displayed a sheet-like proliferation of monotonous oval to round 
Table 1 Summary of the clinicopathologic and molecular features: index series of eight dedifferentiated endometrial carcinomas

\begin{tabular}{|c|c|c|c|c|c|c|c|c|}
\hline & $\begin{array}{c}\text { Age } \\
\text { (years) }\end{array}$ & Histology & Stage & $\begin{array}{l}\text { Mismatch } \\
\text { repair } \\
\text { protein }\end{array}$ & $\begin{array}{l}\text { Mutations in } \\
\text { endometrioid component }\end{array}$ & $\begin{array}{l}\text { Mutations in } \\
\text { undifferentiated component }\end{array}$ & BRG1/ BRM & INI1/ BRM \\
\hline 1 & 54 & $\begin{array}{c}90 \% \\
\text { Endometrioid } \\
\text { grade } 1 ; 10 \% \\
\text { undifferentiated }\end{array}$ & $1 \mathrm{~A}$ & Intact & & $\begin{array}{l}\text { MSH6 (A25S), PIK3R1 } \\
\text { (K567E, R461X), PIK3R2 } \\
\text { (R574Q), POLE (M1L, S459F), } \\
\text { PTEN (R130L, S59X), SPOP } \\
\text { (D140N) }\end{array}$ & $\begin{array}{l}\text { Endometrioid: } \\
\text { intact; } \\
\text { undifferentiated: } \\
\text { intact }\end{array}$ & $\begin{array}{l}\text { Endometrioid: } \\
\text { intact; } \\
\text { undifferentiated: } \\
\text { deficient }\end{array}$ \\
\hline 2 & 55 & $\begin{array}{l}20 \% \\
\text { Endometrioid } \\
\text { grade } 2 ; 80 \% \\
\text { undifferentiated }\end{array}$ & 3B & $\begin{array}{l}\text { MLH1 and } \\
\text { PMS2 loss }\end{array}$ & $\begin{array}{l}\text { ARID1A (f.s.X3, R1749K), } \\
\text { CTNNB1 (S33A), FBXW7 } \\
\text { (A423T), JAK1 (f.s.), } \\
\text { PIK3CA (E545K), POLE } \\
\text { (S2231N), PTEN (deletion, } \\
\text { f.s.), RPL22 } \\
\text { (f.s.) }\end{array}$ & $\begin{array}{l}\text { ARID1A (f.s.X3, R1749K), } \\
\text { CTNNB1 (S33A), FBXW7 } \\
\text { (A423T), JAK1 (f.s.), PIK3CA } \\
\text { (E545K), POLE (S2231N), } \\
\text { PTEN (deletion, f.s.), RPL22 } \\
\text { (f.s.) }\end{array}$ & $\begin{array}{l}\text { Endometrioid: } \\
\text { intact; } \\
\text { undifferentiated: } \\
\text { intact }\end{array}$ & $\begin{array}{l}\text { Endometrioid: } \\
\text { intact; } \\
\text { undifferentiated: } \\
\text { intact }\end{array}$ \\
\hline 3 & 70 & $\begin{array}{c}10 \% \\
\text { Endometrioid } \\
\text { grade 1; } 90 \% \\
\text { undifferentiated }\end{array}$ & 3C1 & PMS2 loss & & $\begin{array}{l}\text { ARID1A (f.s.x2), FBXW7 } \\
\text { (R399Q), KRAS (A146T), } \\
\text { PIK3CA (E545A), POLE } \\
\text { (R573L), PTEN (f.s.x2, } \\
\text { L139X), SMARCA4 } \\
\text { (f.s. C1046R) }\end{array}$ & $\begin{array}{l}\text { Endometrioid: } \\
\text { intact; } \\
\text { undifferentiated: } \\
\text { deficient }\end{array}$ & $\begin{array}{l}\text { Endometrioid: } \\
\text { intact; } \\
\text { undifferentiated: } \\
\text { intact }\end{array}$ \\
\hline 4 & 73 & $\begin{array}{c}10 \% \\
\text { Endometrioid } \\
\text { grade } 1 ; 90 \% \\
\text { undifferentiated }\end{array}$ & $1 \mathrm{~A}$ & $\begin{array}{l}\text { MLH1 and } \\
\text { PMS2 loss }\end{array}$ & & $\begin{array}{l}\text { ARID1A (W1498X), KRAS } \\
\text { (G12A), PIK3CA (E545K, } \\
\text { V344M), PTEN (K128N), } \\
\text { SMARCA4 } \\
\text { (f.s., Q756X), SPOP (E50K) }\end{array}$ & $\begin{array}{l}\text { Endometrioid: } \\
\text { intact; } \\
\text { undifferentiated: } \\
\text { deficient }\end{array}$ & $\begin{array}{l}\text { Endometrioid: } \\
\text { intact; } \\
\text { undifferentiated: } \\
\text { intact }\end{array}$ \\
\hline 5 & 66 & $\begin{array}{c}70 \% \\
\text { Endometrioid } \\
\text { grade } 1 ; 30 \% \\
\text { undifferentiated }\end{array}$ & $1 \mathrm{~B}$ & PMS2 loss & $\begin{array}{l}\text { ARID1A (f.s., N102S), } \\
\text { CTNNB1 (T41A), JAK1 } \\
\text { (f.s.X2), MSH2 (f.s.), PIK3R1 } \\
\text { (f.s.), PTEN (f.s.), RPL22 (f. } \\
\text { s.), SMARCA4 (P327S) }\end{array}$ & $\begin{array}{l}\text { ARID1A (f.s.), JAK1 (f.s.X2), } \\
\text { KRAS (G12D), MSH2 (f.s.), } \\
\text { PTEN (f.s.), RPL22 (f.s.), } \\
\text { SMARCA4 (f.s.x2, R1203C) }\end{array}$ & $\begin{array}{l}\text { Endometrioid: } \\
\text { intact; } \\
\text { undifferentiated: } \\
\text { deficient }\end{array}$ & $\begin{array}{l}\text { Endometrioid: } \\
\text { intact; } \\
\text { undifferentiated: } \\
\text { intact }\end{array}$ \\
\hline 6 & 73 & $\begin{array}{c}30 \% \\
\text { Endometrioid } \\
\text { grade 1, 70\% } \\
\text { undifferentiated }\end{array}$ & $1 \mathrm{~B}$ & $\begin{array}{l}\text { MLH1 and } \\
\text { PMS2 loss }\end{array}$ & $\begin{array}{l}\text { FBXW7 (deletion), HRAS } \\
\text { (R68W), JAK1 (f.s., Y412X), } \\
\text { PIK3CA (N575K, H1047L), } \\
\text { PTEN (Y68X, } \\
\text { f.s.), PPP2R1A (R418Q) }\end{array}$ & $\begin{array}{l}\text { FBXW7 (R49P), JAK1 } \\
\text { (f.s., Y412X), PIK3CA } \\
\text { (N575K, H1047L), PTEN } \\
\text { (Y68X, f.s.), PPP2R1A } \\
\text { (R418Q) }\end{array}$ & $\begin{array}{l}\text { Endometrioid: } \\
\text { intact; } \\
\text { undifferentiated: } \\
\text { intact }\end{array}$ & $\begin{array}{l}\text { Endometrioid: } \\
\text { intact; } \\
\text { undifferentiated: } \\
\text { intact }\end{array}$ \\
\hline 7 & 51 & $\begin{array}{c}60 \% \\
\text { Endometrioid } \\
\text { grade } 1 ; 40 \% \\
\text { undifferentiated }\end{array}$ & $3 \mathrm{C} 2$ & $\begin{array}{l}\text { MLH1 and } \\
\text { PMS2 loss }\end{array}$ & $\begin{array}{l}\text { ARID1A (f.s.), JAK1 (f.s.), } \\
\text { KRAS (G12V), PIK3R1 } \\
\text { (deletion), PTEN (f.s.), } \\
\text { RNF43 (f.s.), RPL22 (f.s.) }\end{array}$ & $\begin{array}{l}\text { ARID1A (f.s.), JAK1 (f.s.), } \\
\text { KRAS (G12V), PIK3R1 } \\
\text { (deletion), POLE (R1284Q), } \\
\text { PTEN (f.s.), RNF43 (f.s.), } \\
\text { RPL22 (f.s.), SMARCA4 (f.s.), } \\
\text { TP53 (R26C) }\end{array}$ & $\begin{array}{l}\text { Endometrioid: } \\
\text { intact; } \\
\text { undifferentiated: } \\
\text { deficient }\end{array}$ & $\begin{array}{l}\text { Endometrioid: } \\
\text { intact; } \\
\text { undifferentiated: } \\
\text { intact }\end{array}$ \\
\hline 8 & 46 & $\begin{array}{c}5 \% \\
\text { Endometrioid } \\
\text { grade } 2 ; 95 \% \\
\text { undifferentiated }\end{array}$ & $1 \mathrm{~A}$ & Intact & & $\begin{array}{l}\text { ARID1A (Q1172X), FGFR2 } \\
\text { (E413D), MSH2 (E580X, } \\
\text { K918N), PIK3CA (R93W, } \\
\text { T1025 A), PIK3R1 (E217K), } \\
\text { PMS2 (R563X, E172A), POLE } \\
\text { (V411L), PTEN (E299X), } \\
\text { SPOP (K101T), SMARCA4 } \\
\text { (R549C) }\end{array}$ & $\begin{array}{l}\text { Endometrioid: } \\
\text { intact; } \\
\text { undifferentiated: } \\
\text { intact }\end{array}$ & $\begin{array}{l}\text { Endometrioid: } \\
\text { intact; } \\
\text { undifferentiated: } \\
\text { deficient }\end{array}$ \\
\hline
\end{tabular}

Abbreviations: BRG1, protein encoded by SMARCA4; BRM, protein encoded by SMARCA2; FIGO, The international federation of gynecology and obstetrics; f.s., frameshift mutation; INI1, protein encoded by SMARCB1; stage, based on 2009 FIGO staging.

* The corresponding endometrioid and the undifferentiated components displayed same mismatch repair protein immunostaining pattern in each individual tumor.

cells in all 10 cases and constituted between 10 and $95 \%$ of the overall tumor. The undifferentiated component all displayed prominent mitotic activity with tumor necrosis. Cellular discohesion was noted in all cases, and rhabdoid cytologic features were seen in seven cases (Figure 1). Six of the eight tumors were mismatch repair-deficient, showing the same pattern of mismatch repair deficiency in the corresponding endometrioid and undifferentiated components.
The mutation profiles of the undifferentiated component in this index series are shown in Table 1. We identified recurrent mutations involving PTEN (8/8), ARID1A (6/8), PIK3CA (5/8), SMARCA4 (5/8, with frameshift/nonsense mutations in 4 cases and a missense mutation in 1 case), JAK1 (4/8), KRAS (4/8), PIK3R1 (4/8), POLE (4/8, only 1 involving exonuclease domain in case 8), RPL22 (3/8), FBXW7 (3/8), SPOP (3/8), and CTNNB1 (2/8). In the four cases where the endometrioid and the 

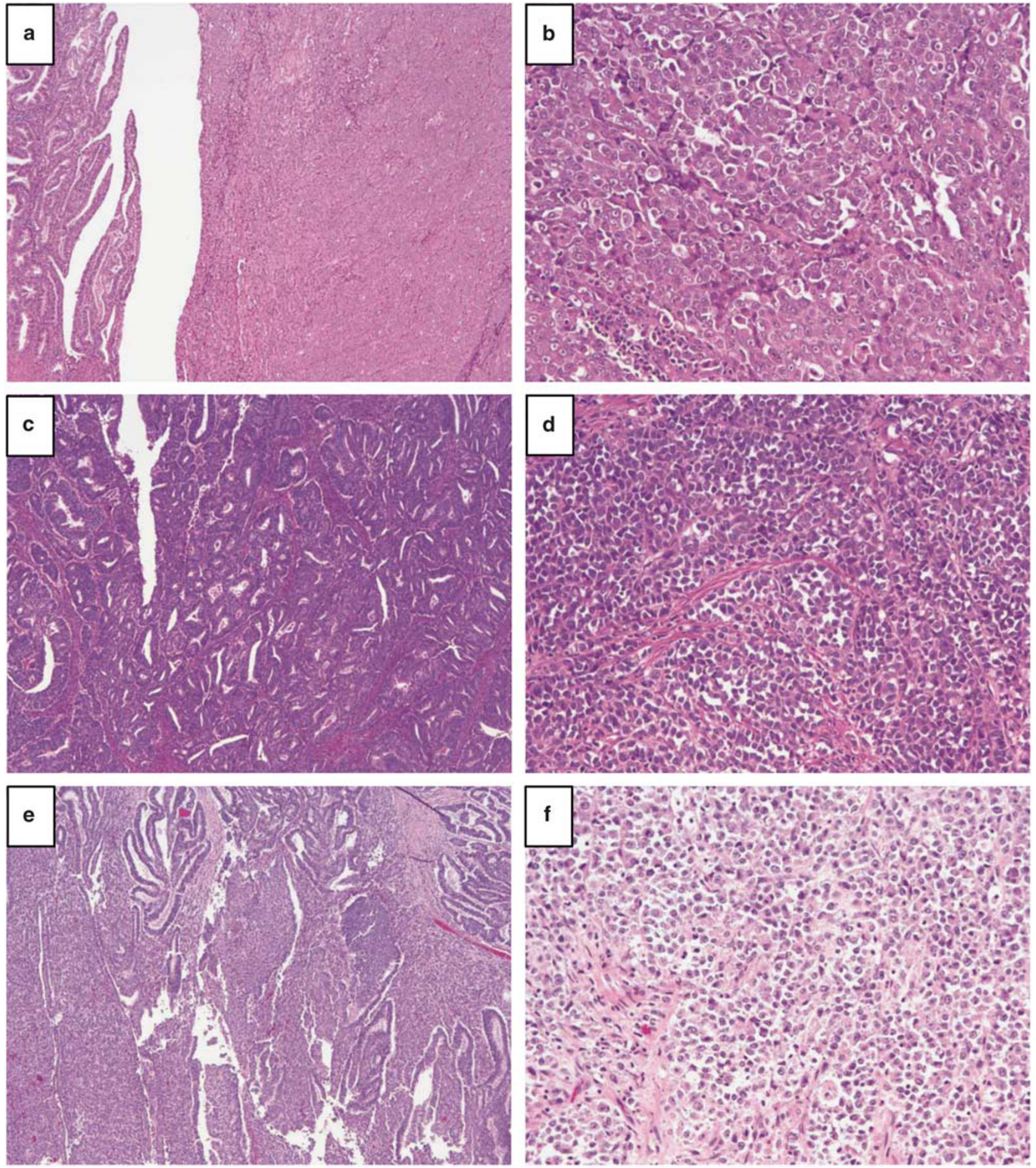

Figure 1 Representative images of dedifferentiated endometrial carcinomas. (a and b) Case 3 showing both the FIGO (The International Federation of Gynecology and Obstetrics) grade 1 endometrioid and the undifferentiated component in (a), with a higher magnification of the undifferentiated component in (b). (c and d) Case 5 showing the FIGO grade 1 endometrioid component in (c) and the undifferentiated component in (d). (e and f) Case 7 showing both the FIGO grade 1 endometrioid and the undifferentiated component in (e), with a higher magnification of the undifferentiated component in (f).

undifferentiated components were both analyzed, $67 \%$ of the mutations identified were shared between the two histologic components. One of the two mismatch repair-intact tumors (case 8) harbored a V411L POLE exonuclease domain mutation. Although the overall mutation profiles are in keeping with the endometrioid histotype, ${ }^{13}$ the findings of frequent frameshift and/or nonsense SMARCA4 
mutations solely in the undifferentiated component warranted further investigation. Four tumors (cases 3, 4, 5, and 7) harbored inactivating SMARCA4 mutations (frameshift and/or nonsense) and all 4 tumors were mismatch repair-deficient. Subsequent Sanger sequencing confirmed the somatic nature of these frameshift/nonsense SMARCA4 mutations.

\section{Immunohistochemical Analysis of SWI/SNF Complex Proteins}

Based on the mutation findings, we examined the expression of the SMARCA4 (BRG1) ATPase as well as two other members of SWI/SNF complex-SMARCB1 (INI1) and SMARCA2 (BRM), the other mutually exclusive ATPase of the SWI/SNF complex-by immunohistochemistry in the index series. All four mismatch repair-deficient tumors harboring inactivating SMARCA4 mutations showed a complete loss of SMARCA4 expression in the undifferentiated component but intact SMARCA4 expression in the corresponding endometrioid component (Figure 2). There is concurrent loss of SMARCA2 expression in the undifferentiated component of these four SMARCA4-mutated/ deficient tumors, whereas SMARCB1 expression was intact in these four tumors. The three tumors (cases 1,2, and 6) with wild-type SMARCA4 and the POLE (V411L)-mutated tumor (case 8) with a missense mutation (R549C) in SMARCA4 mutation all showed intact SMARCA4 expression in both the endometrioid and the undifferentiated components. Two of these SMARCA4-intact tumors (cases 1 and 8) showed a complete loss of SMARCB1 and SMARCA2 expression in the undifferentiated component but intact SMARCB1 and SMARCA2 expression in the corresponding endometrioid component (Figure 3), whereas the remaining two SMARCA4-intact tumors (cases 2 and 6) showed intact SMARCB1 and SMARCA2 expression throughout the tumor (Figure 4).

To further validate these results and determine the frequency of SWI/SNF protein loss in dedifferentiated endometrial carcinoma, we surveyed their expression in an additional series of 22 centrally reviewed dedifferentiated endometrial carcinomas. The undifferentiated component constituted on average $60 \%$ of the overall tumor (ranging from 5 to $95 \%$ ). Seventy percent of the tumors were mismatch repair-deficient, with the corresponding endometrioid and undifferentiated components in these mismatch repair-deficient tumors showing the same pattern of mismatch repair protein loss (Table 2). We also included a series of 31 FIGO grade 3 endometrioid carcinomas for comparison; these 31 cases were all initially diagnosed as dedifferentiated endometrial carcinomas but were reassigned to FIGO grade 3 endometrioid carcinomas upon centralized review. The reasons for the histologic reassignment were the identification of focal glandular differentiation and/or the presence of well-demarcated cohesive lobules-features that are not in keeping with the diagnosis of dedifferentiated endometrial carcinoma (Figures $4 \mathrm{e}$ and f). In comparison, $63 \%$ (19 of 30 cases with interpretable results) of the tumors reclassified as FIGO grade 3 endometrioid carcinomas were mismatch repairdeficient (Table 2).

In the validation cohort, 7 of the 22 dedifferentiated carcinomas showed a complete loss of SMARCA2 and SMARCA4 in the undifferentiated component, whereas the corresponding endometrioid component showed intact expression. SMARCB1 expression was intact in these SMARCA4-deficient tumors. Of the remaining 15 SMARCA4-intact tumors, 2 showed a complete loss of SMARCA2 and SMARCB1 in the undifferentiated component and intact expression in the corresponding endometrioid component. These findings confirm the frequent loss of SMARCA2, SMARCA4, and SMARCB1 in the undifferentiated component of dedifferentiated endometrial carcinomas. In comparison, none of the 31 FIGO grade 3 endometrioid carcinomas examined showed SMARCA2, SMARCA4, and/or SMARCB1 loss.

\section{Clinicopathologic Features of SWI/SNF-Deficient Dedifferentiated Endometrial Carcinomas}

Combining our findings in the index series and the validation series (Table 2), SMARCA4/SMARCA2 deficiency in the undifferentiated component was found in 11/30 dedifferentiated carcinomas (37\%) and SMARCB1/SMARCA2 deficiency in 4/30 (13\%). The loss of SMARCA4 or SMARCB1 was mutually exclusive, thus loss of either protein was observed in 15/30 (50\%) dedifferentiated carcinomas overall, whereas the loss of SMARCA2 was observed whenever SMARCA4 loss or SMARCAB1 loss occurred. Eight of eleven (73\%) SMARCA4/SMARCA2-deficient tumors were mismatch repair-deficient, whereas 2 of $4(50 \%)$ SMARCB1/SMARCA2-deficient tumors and 11 of 15 $(73 \%)$ of SWI/SNF-intact tumors were mismatch repair-deficient. Histologically, the undifferentiated component of SWI/SNF-deficient dedifferentiated carcinomas was the predominant component in $67 \%$ of the cases (ranging from 5 to 95\%). The differentiated component in all 15 SWI/SNF-deficient dedifferentiated carcinomas exhibited typical FIGO grade 1 or 2 endometrioid morphology. The undifferentiated components all showed sheet-like proliferation of monomorphic round to polygonal-shaped cells with a vascular stroma (fine capillary network). There were patchy foci (readily identified by low-power examination) of cellular discohesion and focal rhabdoid cytologic features in all cases. Tumor necrosis was present in all cases. Mitotic activity was high in the undifferentiated component, with an average mitotic rate of $49 \mathrm{MF} / 10$ high-power fields $\left(1.5 \mathrm{~mm}^{2}\right)$ that ranged from 35 to $65 \mathrm{MF} / 10$ 
high-power fields. There was no abrupt keratinization noted in any of the cases. Overall, the histologic appearance of the SWI/SNF-deficient dedifferentiated tumors was similar to that of SWI/SNF-intact dedifferentiated tumors (Figures 2,3, and 4$)$.
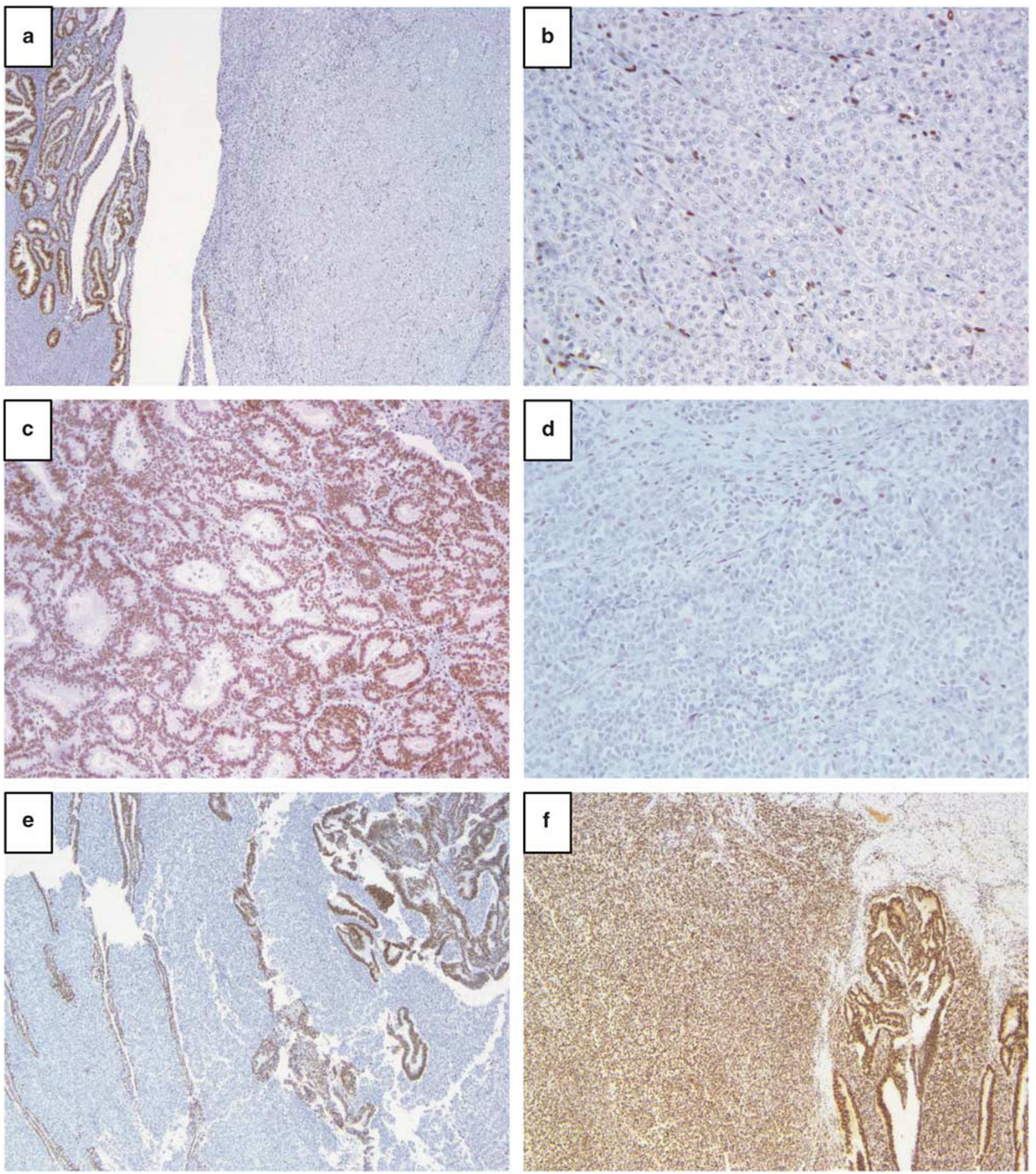

Figure 2 SMARCA4 (BRG1) and SMARCB1 (INI1) immunostaining in SMARCA4/SMARCA2-deficient dedifferentiated endometrial carcinomas. (a and b) Case 3 showing a complete loss of SMARCA4 nuclear staining in the undifferentiated component (with internal stromal positive control) and intact SMARCA4 nuclear staining in the differentiated endometrioid component. (c and d) Case 3 showing a complete loss of SMARCA4 nuclear staining in the undifferentiated component (with internal stromal positive control) and intact SMARCA4 nuclear staining in the differentiated endometrioid component. (e) Case 7 showing a complete loss of SMARCA4 nuclear staining in the undifferentiated component and intact SMARCA4 nuclear staining in the differentiated endometrioid component. (f) Case 7 showing intact SMARCB1 nuclear staining in both the differentiated endometrioid component and the undifferentiated component. 
Clinically, the average age at the time of disease diagnosis was 61 years (ranging from 46 to 75 years) for patients with SWI/SNF-deficient dedifferentiated carcinoma, 62 years (ranging from 39 to 93 years) for patients with SWI/SNF-intact dedifferentiated carcinoma, and 61 years (ranging from 34 to 86 years) for patients with grade 3 endometrioid carcinomas. In terms of FIGO stage, $47 \%$ of the
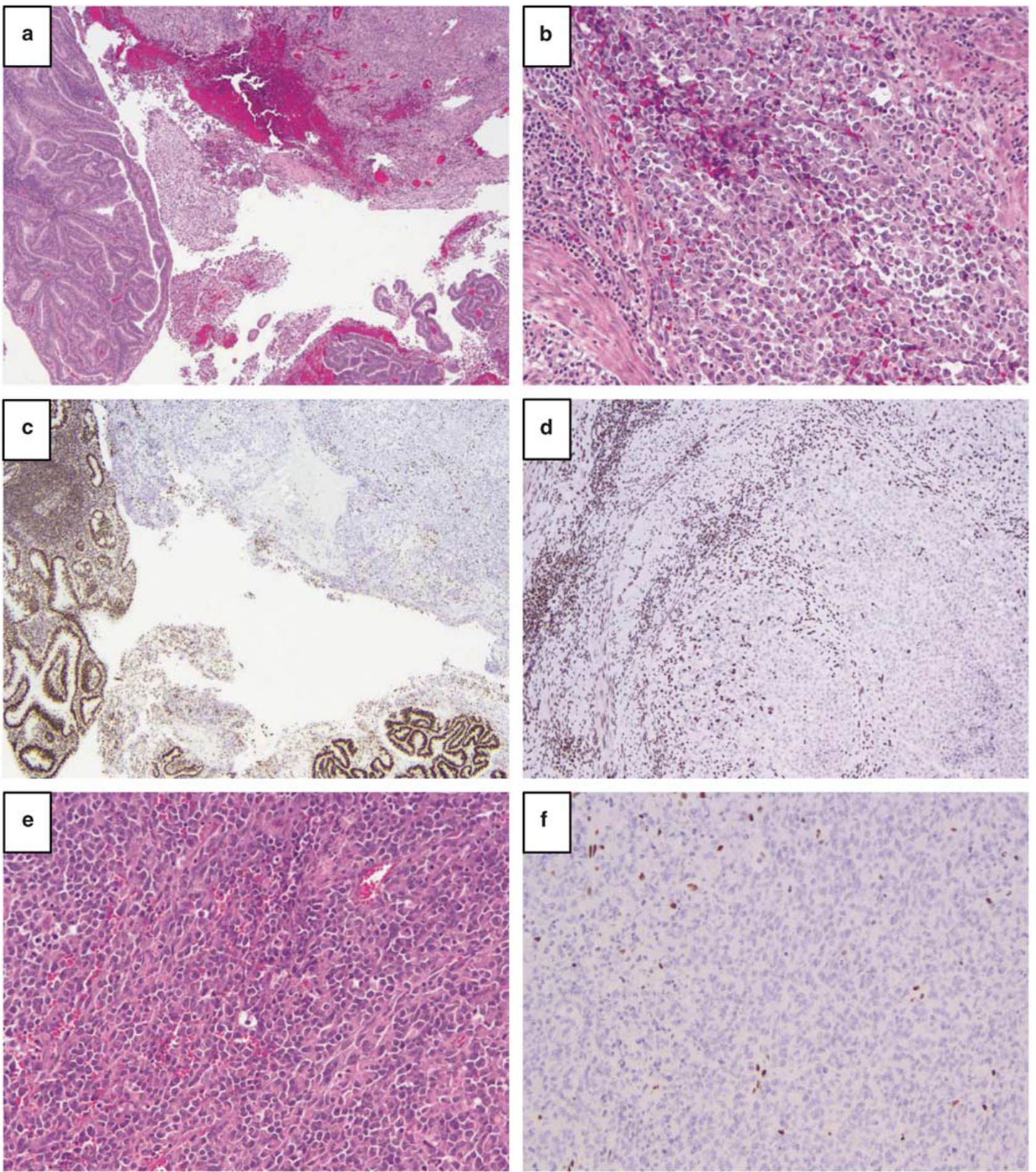

Figure 3 SMARCB1 (INI1) immunostaining in SMARCB1/SMARCA2-deficient dedifferentiated endometrial carcinomas. (a and b) Case 1 showing FIGO (The International Federation of Gynecology and Obstetrics) grade 1 endometrioid component and undifferentiated component in (a), with a higher magnification of the undifferentiated component in (b). (c and d) Case 1 showing a complete loss of SMARCB1 nuclear staining in the undifferentiated component (with internal stromal positive control) and intact SMARCB1 nuclear staining in the differentiated endometrioid component. (e and f) Case 8 showing SMARCB1-deficient undifferentiated component. 
patients with SWI/SNF-deficient dedifferentiated carcinoma had stage 2-4 disease, compared with $33 \%$ of the patients with SWI/SNF-intact dedifferentiated carcinoma and $23 \%$ of patients with grade 3 endometrioid carcinoma. In terms of adjuvant treatment, $70 \%$ of the patients with dedifferentiated carcinoma received adjuvant therapy (radiation therapy and/or chemotherapy),
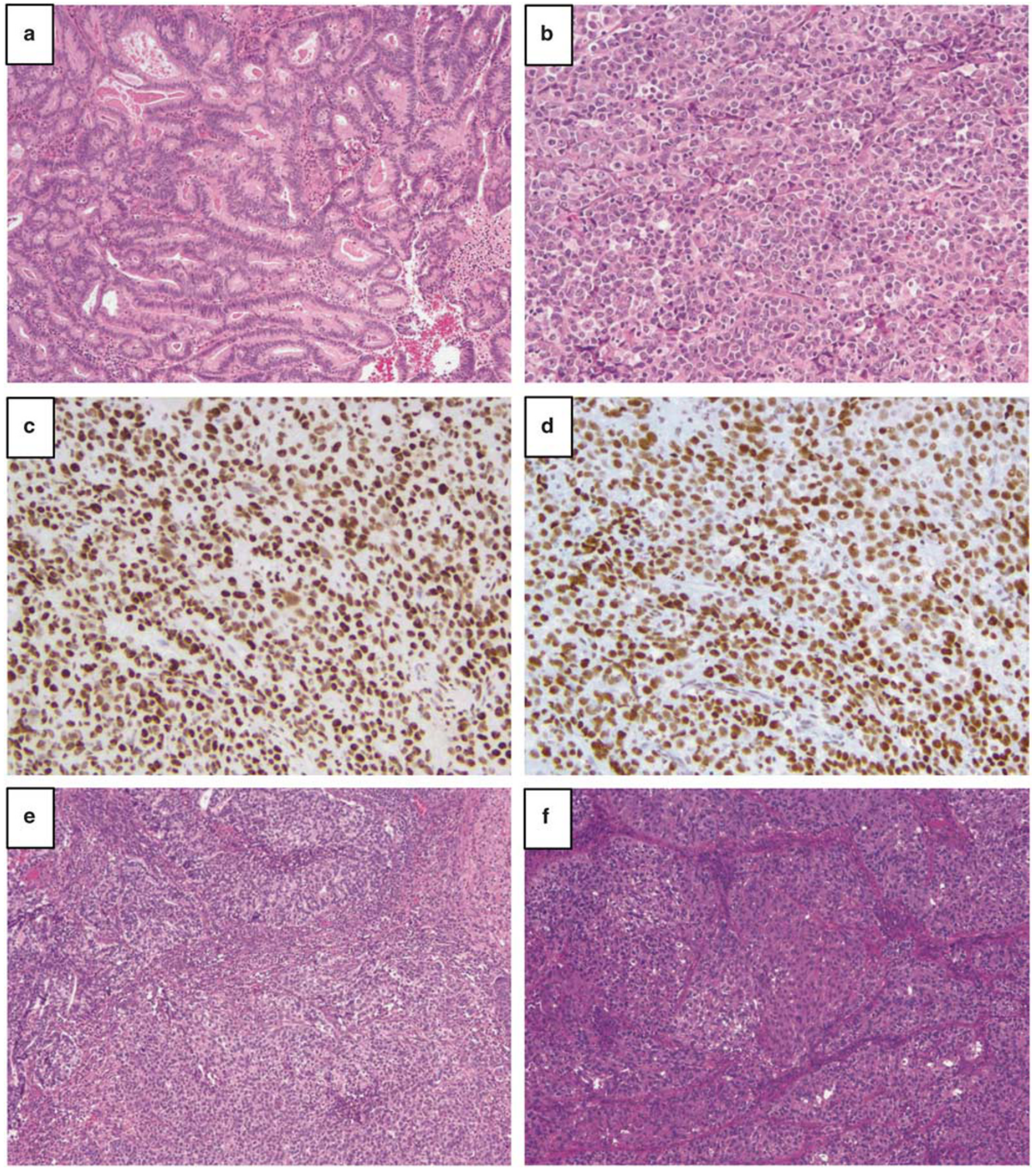

Figure 4 SWI/SNF-intact dedifferentiated endometrial carcinoma and FIGO (The International Federation of Gynecology and Obstetrics) grade 3 endometrioid carcinomas. Case 2 showing the low-grade endometrioid component (a) and undifferentiated component (b), with intact SMARCA4 (c) and intact SMARCB1 (d) expression. (e and f) Two cases of FIGO grade 3 endometrioid carcinomas, with one case showing focal glandular differentiation and the lack of cellular discohesion (e) and the other case showing well-demarcated cohesive lobules (f). 
Table 2 Summary of SWI/SNF and mismatch repair protein immunohistochemistry results in the entire study cohort

\begin{tabular}{|c|c|c|c|}
\hline & $\begin{array}{l}\text { SWI/SNF } \\
\text { status }\end{array}$ & $\begin{array}{l}\text { Frequency of mismatch repair } \\
\text { deficiency }\end{array}$ & Details of mismatch repair deficiency \\
\hline $\begin{array}{l}\text { Dedifferentiated endometrial } \\
\text { carcinoma }(\mathrm{n}=30)\end{array}$ & & $21(70 \%)$ & \\
\hline SMARCA4/A2-deficient & $11(37 \%)$ & $8(73 \%)$ & 5 MLH1/PMS2 - ; 3 PMS2- \\
\hline SMARCB1/A2-deficient & $4(13 \%)$ & $2(50 \%)$ & 2 MLH1/PMS2 - \\
\hline SMARCA4/A2/B1-intact & $15(50 \%)$ & $11(73 \%)$ & $\begin{array}{c}6 \text { MLH1/PMS2 - ; } 2 \text { PMS2 - ; } 2 \text { MSH2/MSH6 - ; } \\
1 \text { MSH6 - }\end{array}$ \\
\hline $\begin{array}{l}\text { FIGO grade } 3 \text { endometrioid carcinoma } \\
(\mathrm{n}=31)\end{array}$ & & $19(63 \%)^{\mathrm{a}}$ & \\
\hline SMARCA4/A2-deficient & $0(0 \%)$ & & \\
\hline SMARCB1/A2-deficient & $0(0 \%)$ & & \\
\hline SMARCA4/A2/B1-intact & $31(100 \%)$ & $19(63 \%)^{\mathrm{a}}$ & $\begin{array}{c}13 \text { MLH1/PMS2 - ; } 2 \text { PMS2 - ; } 2 \text { MSH2/ } \\
\text { MSH6 - ; } 2 \text { MSH6 - }\end{array}$ \\
\hline
\end{tabular}

${ }^{\mathrm{a}}$ Out of 30 cases with interpretable mismatch repair protein immunostaining results.

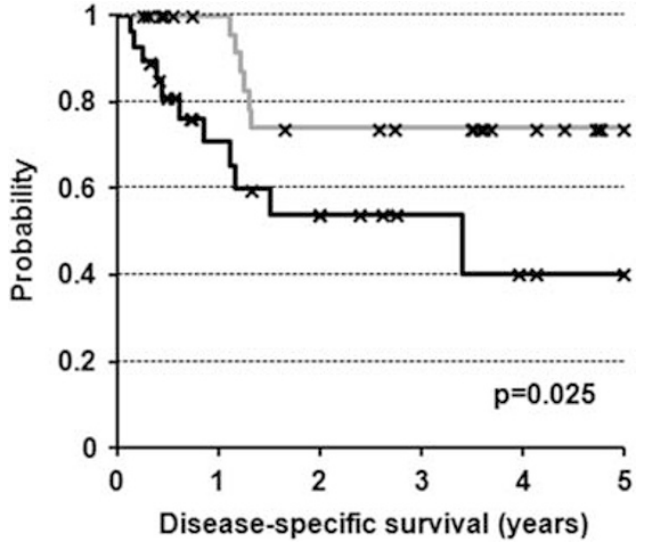

Figure 5 Kaplan-Meier survival analysis: Decreased diseasespecific survival in review-confirmed dedifferentiated endometrial carcinomas (black solid line; $n=30$ ) compared with FIGO (The International Federation of Gynecology and Obstetrics) grade 3 endometrioid carcinomas (gray solid line; $n=31$ ) that were initially diagnosed as dedifferentiated carcinoma.

whereas $22 \%$ of the patients declined adjuvant therapy, compared with $83 \%$ of the patients with grade 3 endometrioid carcinoma that received and $7 \%$ that declined adjuvant therapy. With respect to patient outcome, dedifferentiated carcinoma as a group had a worse disease-specific survival compared with FIGO grade 3 endometrioid carcinoma $(P=0.025)$ (Figure 5). There was no significant difference with respect to disease-specific survival between SWI/SNF-deficient dedifferentiated carcinomas and SWI/SNF-intact dedifferentiated carcinomas $(P=0.92)$ based on Kaplan-Meier survival analysis (data not shown).

\section{Discussion}

Dedifferentiated endometrial carcinoma is a morphologically distinct subtype of endometrial carcinoma. As demonstrated by earlier studies and by our current study, ${ }^{4,5}$ the undifferentiated component shares the same molecular aberrations as the corresponding endometrioid component. In contrast to the differentiated endometrioid component that displays clear evidence of epithelial differentiation (in the form of glandular or villoglandular structures), the undifferentiated component lacks any histologic evidence of glandular/villoglandular differentiation. This corresponds to the absence or markedly reduced expression of epithelial markers (ie, cytokeratins and epithelial membrane antigen) in the undifferentiated component compared with the endometrioid component. $^{2}$

In this study, we identified through targeted sequencing and subsequent immunohistochemical validation that concurrent loss of SMARCA4/SMARCA2 or concurrent loss of SMARCB1/SMARCA2 expression is associated with histologic dedifferentiation in half of dedifferentiated endometrial carcinomas. The findings of SMARCA4-inactivating mutations (frameshift and/or nonsense) in the undifferentiated component but not in the differentiated endometrioid component further support the hypothesis that undifferentiated component represents a distinct form of tumor progression from the pre-existing low-grade endometrioid carcinoma. Our immunohistochemical findings corroborate the recent reports by Strehl et a ${ }^{14}$ and by Stewart and Crook $^{15}$ that demonstrated the loss of SMARCA4/SMARCB1 expression in the undifferentiated component of a subset of dedifferentiated endometrial carcinoma. Strehl et $a l^{14}$ described a single case of SMARCA4-deficient dedifferentiated endometrial carcinoma comprised of endometrioid component and an undifferentiated component with prominent rhabdoid cytologic features. That case showed loss of SMARCA4 expression by immunohistochemistry in the undifferentiated component with intact SMARCA4 expression in the endometrioid component. SMARCB1 and mismatch repair protein expression were intact in both components in this case. Strehl et al ${ }^{14}$ also surveyed the expression of SMARCA4 and SMARCB1 in 24 FIGO grade 3 endometrioid carcinomas and identified no loss of expression in these tumors, similar to our 
findings here. Stewart and Crook $^{15}$ examined the expression of SMARCA4, SMARCB1, and mismatch repair proteins in 17 dedifferentiated endometrial carcinomas. They observed a loss of SMARCA4 that was limited only to the undifferentiated component in 3 of $17(18 \%)$ tumors and a loss of SMARCB1 in both the endometrioid and undifferentiated components in 1 of $17(6 \%)$ tumors. In the present study, we observed a higher frequency of SMARCA4 loss at 37\% and SMARCB1 loss at $13 \%$ in our cohort. Even though there is a difference in the sample size between the two studies, we speculate that our histologic inclusion criteria may account for at least some of the differences here. We closely adhered to the original histological description in our centralized histologic review, in which we reclassified about half of the cases originally diagnosed as dedifferentiated endometrial carcinomas to FIGO grade 3 endometrioid carcinomas. For instance, we did not include tumors that displayed spindled epithelial component or cohesive solid architecture in the undifferentiated component. The importance of this centralized review is further underscored by our findings that review-confirmed dedifferentiated endometrial carcinomas showed worse disease-specific survival than tumors reclassified as FIGO grade 3 endometrioid carcinomas. This raises an important diagnostic issue with regard to the subjectivity in the diagnosis of dedifferentiated endometrial carcinoma, even among subspecialty gynecologic pathologists, and the need for more objective criteria through better molecular understanding. Although core SWI/SNF protein deficiency as demonstrated by immunohistochemistry may help to confirm the diagnosis of dedifferentiated endometrial carcinoma in the appropriate histologic context, intact expression of SMARCA2, SMARCA4, and SMARCB1 does not exclude the possibility of dedifferentiated endometrial carcinoma. Furthermore, it is important to note that an abnormal mismatch repair protein immunostaining result does not necessarily support the diagnosis of dedifferentiated endometrial carcinomas, as we observed frequent $(63 \%)$ mismatch repair deficiency in FIGO grade 3 endometrioid carcinomas that were initially diagnosed as dedifferentiated endometrial carcinomas, which is slightly higher than the $45 \%$ recently reported for typical FIGO grade 3 endometrioid carcinomas. ${ }^{16}$

SMARCA2, SMARCA4, and SMARCB1 are all core components of an evolutionarily onserved multi-subunit SWI/SNF chromatin remodeling complex. SWI/SNF complex has an important role in several cellular processes such as transcription, cell differentiation, and DNA repair. ${ }^{17,18}$ Mutations in the SWI/SNF chromatin remodeling complex have been reported in up to $20 \%$ of human cancers. ${ }^{17,19}$ Whereas non-core SWI/SNF members such as $A R I D 1 A$ are mutated in common tumors such as endometrioid carcinomas of the gynecologic tract and gastric adenocarcinomas, ${ }^{20-22}$ loss of one or more core SWI/SNF members occurs in several rare tumor types such as rhabdoid tumors of the brain, kidney, and extrarenal sites, ${ }^{23}$ epithelioid sarcoma, ${ }^{24,25}$ and small-cell carcinoma of the ovary-hypercalcemic type, ${ }^{12,26-30}$ as well as in a subset of pancreatic undifferentiated carcinoma and a subset of poorly differentiated carcinomas of the sinonasal tract. ${ }^{31,32}$ The central role of the SWI/SNF complex in transcriptional regulation suggests that the principle mechanism of oncogenesis upon mutation of its components is likely epigenetic dysregulation. The common pathological features among several tumors lacking combinations of SMARCA2, SMARCA4, and/or SMARCB1-sheetlike growth of undifferentiated, highly proliferative cells with cytology ranging from small round cell to rhabdoid-imply that core SWI/SNF protein deficiency in these aggressive tumors may prevent the activation of genes that promote terminal differentiation and cell-cell adhesion. Consistent with this hypothesis, the undifferentiated component of dedifferentiated endometrial carcinoma show absent or reduced expression of differentiation markers that are present in the well-differentiated areas. ${ }^{2,5,14,15}$ Similarly, renal cell carcinoma with rhabdoid morphology, a tumor that shows combined core SWI/SNF protein deficiency (SMARCA4 and SMARCB1), has also been proposed to be a dedifferentiated tumor. ${ }^{33}$ In lung cancer, loss of SMARCA4 and SMARCA2 proteins is associated with poorly differentiated morphology (solid-predominant), reduced bronchial epithelial immunophenotype, and more aggressive disease behavior. ${ }^{34,35}$ Although future studies are needed to provide more mechanistic insights, it is plausible that combined deficiency in core members of the SWI/SNF complex (ie, SMARCA2, SMARCA4, and/or SMARCB1) predisposes to cellular dedifferentiation (in either a susceptible normal cell or a pre-existing low-grade tumor cell), resulting in an aggressive, monomorphic, high-grade tumor with undifferentiated morphology. In terms of therapeutic opportunities, the loss of these core SWI/SNF components such as SMARCA2, SMARCA4, and SMARCB1 in dedifferentiated endometrial carcinoma may render the tumor susceptible to an emerging class of drugs that regulates epigenetic modifications. ${ }^{36,37}$

We observed a high frequency of mismatch repair deficiency in our present series of dedifferentiated endometrial carcinomas, including cases showing SWI/SNF protein deficiency. It therefore appears that a hypermutating molecular context predisposes to molecular event(s) such as SMARCA4 inactivation that induce dedifferentiation. We also identified in our index series a case of dedifferentiated endometrial carcinoma that harbored a hotspot POLE exonuclease domain mutation. This POLE-mutated tumor showed intact mismatch repair protein expression and a loss of SMARCB1 in the undifferentiated component of the tumor. Given that the great majority of POLE exonuclease domain-mutated endometrial carcinomas are associated with a favorable clinical course, ${ }^{13,38,39}$ 
it is unclear whether the observed histologic dedifferentiation that is accompanied by the loss of core SWI/SNF complex proteins would confer more aggressive biology and clinical behavior or whether the typically prognostically favorable ultramutated POLE exonuclease domain-mutated molecular context dominates.

Although the sequencing analysis in our index series showed that frameshift/nonsense SMARCA4 mutation results in the loss of SMARCA4 protein expression in dedifferentiated endometrial sarcomas, we did not interrogate the exact mechanisms underlying the loss of SMARCA2 and SMARCB1 expression in this study. Extrapolating the knowledge we have gained from other tumor types, SMARCB1 protein loss typically occurs as a result of genomic mutation, most frequently through homozygous deletion. ${ }^{40,41}$ Unlike SMARCA4 and SMARCB1, mutations involving SMARCA2 do not appear to be frequent in human cancer. In ovarian small-cell carcinoma-hypercalcemic type, we also observed the same pattern of concurrent SMARCA4/SMARCA2 loss in the great majority of the cases, with a minor subset showing concurrent SMARCA4/SMARCB1 loss. ${ }^{12}$ We found that SMARCA2 protein inactivation was not due to genomic mutation, but instead it appears to result from histone deacetylase-mediated epigenetic silencing of the SMARCA2 gene in SCCOHT, or, alternatively, by an indirect inhibitory effect on SMARCA2 mRNA degradation. ${ }^{42}$ Future studies are, however, needed to delineate precisely the mechanism of SMARCA2 and SMARCB1 inactivation in dedifferentiated endometrial carcinoma.

In summary, we found that loss of either SMARCA4 (by inactivating SMARCA4 mutations) or SMARCB1, together with concurrent loss of SMARCA2, is associated with histologic dedifferentiation in about half of dedifferentiated endometrial carcinoma in the present cohort. These findings suggest that in the SWI/SNF-deficient cases, the coinactivation of SMARCA4/SMARCA2 or SMARCB1/SMARCA2 may be involved in the development of undifferentiated carcinoma from an underlying low-grade endometrioid carcinoma. Further studies are needed to examine the effects of SMARCA4/SMARCB1/SMARCA2 inactivation in the context of endometrioid carcinoma.

\section{Acknowledgments}

This study is supported by a research funds from Cancer Research Society of Canada, Royal Alexandra Hospital foundation, Alberta Cancer Foundation, Calgary Laboratory Services Internal Research Competition (RS10-536).

\section{Disclosure/conflict of interest}

The authors declare no conflict of interest.

\section{References}

1 Silva EG, Deavers MT, Bodurka DC et al. Association of low-grade endometrioid carcinoma of the uterus and ovary with undifferentiated carcinoma: a new type of dedifferentiated carcinoma? Int J Gynecol Pathol 2006;25:52-58.

2 Silva EG, Deavers MT, Malpica A. Undifferentiated carcinoma of the endometrium: a review. Pathology 2007;39:134-138.

3 Giordano G, D'Adda T, Bottarelli L et al. Two cases of low-grade endometriod carcinoma associated with undifferentiated carcinoma of the uterus (dedifferentiated carcinoma): a molecular study. Pathol Oncol Res 2012;18:523-528.

4 Kuhn E, Ayhan A, Bahadirli-Talbott A et al. Molecular characterization of undifferentiated carcinoma associated with endometrioid carcinoma. Am J Surg Pathol 2014;38:660-665.

5 Tafe LJ, Garg K, Chew I et al. Endometrial and ovarian carcinomas with undifferentiated components: clinically aggressive and frequently underrecognized neoplasms. Mod Pathol 2010;23:781-789.

6 Vita G, Borgia L, Di Giovannantonio L et al. Dedifferentiated endometrioid adenocarcinoma of the uterus: a clinicopathologic study of a case. Int J Surg Pathol 2011;19:649-652.

7 Garg K, Leitao MM Jr, Kauff ND et al. Selection of endometrial carcinomas for DNA mismatch repair protein immunohistochemistry using patient age and tumor morphology enhances detection of mismatch repair abnormalities. Am J Surg Pathol 2009;33:925-933.

8 Garg K, Shih K, Barakat R et al. Endometrial carcinomas in women aged 40 years and younger: tumors associated with loss of DNA mismatch repair proteins comprise a distinct clinicopathologic subset. Am J Surg Pathol 2009;33:1869-1877.

9 Romero-Perez L, Lopez-Garcia MA, Diaz-Martin J et al. ZEB1 overexpression associated with E-cadherin and microRNA-200 downregulation is characteristic of undifferentiated endometrial carcinoma. Mod Pathol 2013;26:1514-1524.

10 Hoang LN, McConechy MK, Meng B et al. Targeted mutation analysis of endometrial clear cell carcinoma. Histopathology 2015;66:664-674.

11 Hoang LN, Han G, McConechy M et al. Immunohistochemical characterization of prototypical endometrial clear cell carcinoma-diagnostic utility of HNF-1beta and oestrogen receptor. Histopathology 2014;64: 585-596.

12 Karnezis AN, Wang Y, Ramos P et al. Dual loss of the SWI/SNF complex ATPases SMARCA4/BRG1 and SMARCA2/BRM is highly sensitive and specific for small cell carcinoma of the ovary, hypercalcemic type. J Pathol; advance online publication, 10 September 2015; doi:10.1002/path.4633 [e-pub ahead of print].

13 Kandoth C, Schultz N, Cherniack AD et al. Integrated genomic characterization of endometrial carcinoma. Nature 2013;497:67-73.

14 Strehl JD, Wachter DL, Fiedler J et al. Pattern of SMARCB1 (INI1) and SMARCA4 (BRG1) in poorly differentiated endometrioid adenocarcinoma of the uterus: analysis of a series with emphasis on a novel SMARCA4-deficient dedifferentiated rhabdoid variant. Ann Diagn Pathol 2015;19:198-202.

15 Stewart CJ, Crook ML. SWI/SNF complex deficiency and mismatch repair protein expression in 
undifferentiated and dedifferentiated endometrial carcinoma. Pathology 2015;47:439-445.

16 Nelson GS, Pink A, Lee S et al. MMR deficiency is common in high-grade endometrioid carcinomas and is associated with an unfavorable outcome. Gynecol Oncol 2013;131:309-314.

17 Masliah-Planchon J, Bieche I, Guinebretiere JM et al. SWI/SNF chromatin remodeling and human malignancies. Annu Rev Pathol 2015;10:145-171.

18 Wang X, Haswell JR, Roberts CW. Molecular pathways: SWI/SNF (BAF) complexes are frequently mutated in cancer-mechanisms and potential therapeutic insights. Clin Cancer Res 2014;20:21-27.

19 Kadoch C, Hargreaves DC, Hodges C et al. Proteomic and bioinformatic analysis of mammalian SWI/SNF complexes identifies extensive roles in human malignancy. Nat Genet 2013;45:592-601.

20 Jones S, Wang TL, Shih IeM et al. Frequent mutations of chromatin remodeling gene ARID1A in ovarian clear cell carcinoma. Science 2010;330:228-231.

21 Wiegand KC, Shah SP, Al-Agha OM et al. ARID1A mutations in endometriosis-associated ovarian carcinomas. N Engl J Med 2010;363:1532-1543.

$22 \mathrm{Wu}$ JN, Roberts CW. ARID1A mutations in cancer: another epigenetic tumor suppressor? Cancer Discov 2013;3:35-43.

23 Agaimy A. The expanding family of SMARCB1(INI1)deficient neoplasia: implications of phenotypic, biological, and molecular heterogeneity. Adv Anat Pathol 2014;21:394-410.

24 Hornick JL, Dal Cin P, Fletcher CD. Loss of INI1 expression is characteristic of both conventional and proximal-type epithelioid sarcoma. Am J Surg Pathol 2009;33:542-550.

25 Modena P, Lualdi E, Facchinetti F et al. SMARCB1/ INI1 tumor suppressor gene is frequently inactivated in epithelioid sarcomas. Cancer Res 2005;65:4012-4019.

26 Foulkes WD, Clarke BA, Hasselblatt M et al. No small surprise-small cell carcinoma of the ovary, hypercalcaemic type, is a malignant rhabdoid tumour. J Pathol 2014;233:209-214.

27 Jelinic P, Mueller JJ, Olvera $\mathrm{N}$ et al. Recurrent SMARCA4 mutations in small cell carcinoma of the ovary. Nat Genet 2014;46:424-426.

28 Ramos P, Karnezis AN, Craig DW et al. Small cell carcinoma of the ovary, hypercalcemic type, displays frequent inactivating germline and somatic mutations in SMARCA4. Nat Genet 2014;46:427-429.

29 Witkowski L, Carrot-Zhang J, Albrecht S et al. Germline and somatic SMARCA4 mutations characterize small cell carcinoma of the ovary, hypercalcemic type. Nat Genet 2014;46:438-443.
30 Ramos P, Karnezis AN, Hendricks WP et al. Loss of the tumor suppressor SMARCA4 in small cell carcinoma of the ovary, hypercalcemic type (SCCOHT). Rare Diseases 2014;2 (online publication).

31 Agaimy A, Haller F, Frohnauer J et al. Pancreatic undifferentiated rhabdoid carcinoma: KRAS alterations and SMARCB1 expression status define two subtypes. Mod Pathol 2015;28:248-260.

32 Bishop JA, Antonescu CR, Westra WH. SMARCB1 (INI-1)-deficient carcinomas of the sinonasal tract. Am J Surg Pathol 2014;38:1282-1289.

33 Rao Q, Xia QY, Shen Q et al. Coexistent loss of INI1 and BRG1 expression in a rhabdoid renal cell carcinoma (RCC): implications for a possible role of SWI/SNF complex in the pathogenesis of RCC. Int J Clin Exp Pathol 2014;7:1782-1787.

34 Matsubara D, Kishaba Y, Ishikawa S et al. Lung cancer with loss of BRG1/BRM, shows epithelial mesenchymal transition phenotype and distinct histologic and genetic features. Cancer Sci 2013;104:266-273.

35 Orvis T, Hepperla A, Walter V et al. BRG1/SMARCA4 inactivation promotes non-small cell lung cancer aggressiveness by altering chromatin organization. Cancer Res 2014;74:6486-6498.

36 Fillmore CM, Xu C, Desai PT et al. EZH2 inhibition sensitizes BRG1 and EGFR mutant lung tumours to TopoII inhibitors. Nature 2015;520:239-242.

37 Oike $\mathrm{T}$, Ogiwara $\mathrm{H}$, Tominaga $\mathrm{Y}$ et al. A synthetic lethality-based strategy to treat cancers harboring a genetic deficiency in the chromatin remodeling factor BRG1. Cancer Res 2013;73:5508-5518.

38 Church DN, Stelloo E, Nout RA et al. Prognostic significance of POLE proofreading mutations in endometrial cancer. J Natl Cancer Inst 2014;107:402.

39 Meng B, Hoang LN, McIntyre JB et al. POLE exonuclease domain mutation predicts long progression-free survival in grade 3 endometrioid carcinoma of the endometrium. Gynecol Oncol 2014;134:15-19.

40 Biegel JA, Tan L, Zhang $\mathrm{F}$ et al. Alterations of the hSNF5/INI1 gene in central nervous system atypical teratoid/rhabdoid tumors and renal and extrarenal rhabdoid tumors. Clin Cancer Res 2002;8:3461-3467.

41 Le Loarer F, Zhang L, Fletcher CD et al. Consistent SMARCB1 homozygous deletions in epithelioid sarcoma and in a subset of myoepithelial carcinomas can be reliably detected by FISH in archival material. Genes Chromosomes Cancer 2014;53:475-486.

42 Yamamichi N, Yamamichi-Nishina M, Mizutani T et al. The Brm gene suppressed at the post-transcriptional level in various human cell lines is inducible by transient HDAC inhibitor treatment, which exhibits antioncogenic potential. Oncogene 2005;24:5471-5481. 